REVIEW

\title{
Control of root system architecture by phytohormones and environmental signals in rice
}

\author{
Chen Lin and Margret Sauter \\ Plant Developmental Biology and Plant Physiology, University of Kiel, Am Botanischen Garten 5, 24118 Kiel, Germany
}

\begin{abstract}
Drought and flooding are environmental extremes and major threats to crop production. Water uptake is achieved by plant roots which have to explore new soil spaces to alleviate water deficit during drought or to cope with water excess during flooding. Adaptation of the root system architecture helps plants cope with such extreme conditions and is crucial for plant health and survival. While for dicot plants the well studied model plant Arabidopsis thaliana has provided insight into the genetic and molecular regulation of the root system, less information is available for monocot species, which include the agronomically important cereal crops. Rice (Oryza sativa L.) is a semi-aquatic monocot plant that develops strong tolerance to flooding. Flooding tolerance of rice is closely linked to its adaptive root system. The functional root system of rice is mainly composed of crown roots and is shifted to nodal adventitious roots during flooding which allows rice to maintain oxygen supply to the roots and to survive longer periods of partial submergence as compared with other crops. Likewise, a number of drought-tolerance traits of rice are the result of an altered root system architecture. Hence, the structure of the root system adapts to, both, flooding and drought. Understanding the regulatory mechanisms that control root system adaptation to extreme environments is a key task for scientists to accelerate the breeding efforts for stress-tolerant crops. This review summarizes recently identified genes and molecular mechanisms that regulate root system architecture in rice in response to drought and flooding.
\end{abstract}

\section{ARTICLE HISTORY}

Received 21 June 2019 Accepted 11 August 2019

\section{KEYWORDS}

Rice; adventitious roots; root system architecture; flooding; drought; crown rootless mutants; mao hu zi mutants

\section{Introduction}

The necessity for food production is growing with a worldwide increasing population. The World Bank pointed out that 83 million people in 45 countries were starving in 2017 (Prosekov and Ivanova 2018). A current global problem that urgently needs to be addressed is to satisfy the demand for grain yield in view of climatic changes and in view of less usable water and less culturable land in the future. Among the approaches that are taken, improvement of crop production under adverse and extreme conditions is thought to be effective and reasonable in the present stage (Mickelbart et al. 2015). Extreme weather conditions, causing flooding and drought, are threatening crop growth, eventually resulting in loss of grain yield at harvest. Rice (Oryza sativa L.) is an important cereal crop that provides the main source of food for many people in Asia and Africa. Identifying the crucial traits responsible for stress resistance, and breeding rice varieties with these traits such that plants thrive under extreme conditions, are major tasks for scientists.

Roots are indispensable for autotrophic plants. The functional root system anchors the plant, facilitates water uptake, acquisition of mineral nutrients, and engages in the formation of plant-microorganism communities in the rhizosphere (Orman-Ligeza et al. 2013). Roots are most prone to flooding conditions, and are faced with the necessity to ensure water and nutrient supply under oxygen-deficient conditions that limit ATP synthesis (Sauter 2013). The root system adapts to low oxygen and other abiotic stresses as well as to pathogenic and beneficial organisms. Root system architecture describes the three-dimensional shape and the spatial arrangement of roots in soil. The root system is made up of the primary root, lateral roots and adventitious roots (ARs). The three-dimensional root system architecture is determined by the position of the roots, root numbers, root lengths and the root 
growth angles (Rogers and Benfey 2015). The root system is regulated by both, genetic factors and environmental signals and is continuously adapted to exploit the soil for water and nutrient resources under given and oftentimes changing environmental conditions.

Substantial progress has been made in understanding the development of lateral roots and root growth regulation in the dicot model plant Arabidopsis. In contrast to Arabidopsis, the root system of cereal plants is composed mainly of ARs. Even though some physiological and molecular mechanisms that control lateral and AR development are shared, there are also root type-specific or species-specific differences that reflect the phylogenetic plant origin or the adaptation to an ecological niche. As a monocot model plant with the advantage of a relatively small-sized genome, rice is widely used in molecular-genetic studies. Several rice genomes have been sequenced to date (Wang et al. 2018) providing genomic information that help in the characterization of gene functions and in transcriptome studies. Efficient transformation systems, mutant collections and the generation of introgression lines further aid in functional gene analysis. These tools have led to the identification and functional characterization of a number of genes with a role in root development and growth in recent years (Meng et al. 2019). Nonetheless, the molecular network that regulates AR formation, growth and spatial orientation is still largely unknown. An exciting challenge in the future will be to decipher the molecular control of the root system architecture and dissect functional genes for breeders to improve crop tolerance to biotic and abiotic stress. This review focuses on our current understanding of the regulatory mechanisms and molecular players associated with AR system development in rice. In addition, ideotypes are proposed for root system architecture in response to drought and flooding conditions for the aim of further research.

\section{Types of roots in rice system architecture}

Root systems consist of embryonic and postembryonic roots (Fig. 1). Unlike many dicot plants that rely on the primary root and branching lateral roots, most cereal plants, including rice, wheat and barley, form a dense fibrous root system made up of crown roots and nodal ARs (Rebouillat et al. 2008; Coudert et al. 2010). Rice seeds germinate with a thick embryonic radicle. During seedling development, four or five crown roots grow from the coleoptile node (Fig. 1A). These seedling roots play an important role in exploring and monitoring soil conditions (Sauter 2013). At the postseedling stage, AR primordia develop at the stem nodes to eventually form the mature rice root system. ARs are commonly classified into underground and above ground ARs. It depends on the ecotype and on the environment whether ARs are above or under ground. In general, lowland rice produces more underground ARs and few above ground ARs. Deepwater rice is adapted to grow in areas with fluctuating water levels to which they adapt through stem growth and the development of ARs from above ground stem nodes. In addition, ARs form lateral roots to enlarge the area that is explored by the root system (Fig. 1B). Lateral ARs can be categorized into large lateral and small lateral roots, that display different root lengths (Rebouillat et al. 2008). Hence, the different root types contribute to the root system architecture in rice.

\section{Crown and adventitious root formation and growth in rice is controlled by phytohormones}

Root formation and growth in rice is mediated by phytohormones. Auxin and cytokinins are essential for the de novo genesis of root primordia. But recent reports also describe the involvement of other hormones, such as abscisic acid (ABA) and gibberellic acid (GA), in root formation and growth.
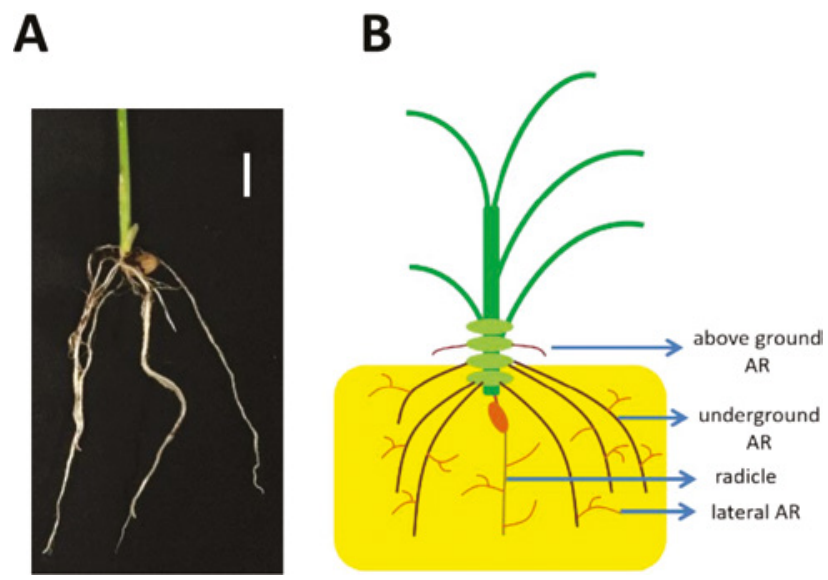

Figure 1. Root types and schematic root system architecture in rice plants. (A) Root system of a 10-day-old rice seedling of the cultured variety Nipponbare (bar $=1 \mathrm{~cm}$ ). (B) Scheme of a mature rice root system in soil. The root system architecture in rice is mainly composed of the radicle with lateral roots, above ground adventitious roots (AR), underground ARs and lateral ARs. 


\section{Auxin}

Previous studies have extensively reported on the role of auxin on the de novo genesis of ARs. In plants, auxin is synthesized mainly through the highly conserved TAA/YUC pathway (Zhao 2012). Indole-3-pyruvate (IPA) is produced by tryptophan (Trp) aminotransferase of Arabidopsis (TAA) from Trp and converted to natural indole-3-acetic acid (IAA) by YUCCA flavin monooxygenases through oxidative decarboxylation (Mashiguchi et al. 2011; Won et al. 2011). Overexpression of OsYUCCA1/OsYUC1 in rice plants results in more crown root formation (Yamamoto et al. 2007; Zhang et al. 2018). Similarly, overexpression of OsYUC3, OsYUC5, OsYUC6, OsYUC7, OsYUC8 and OsYUC11 promotes crown root initiation indicating that the YUCCA gene family universally regulates root formation by controlling the biosynthesis of endogenous auxin. The disruption of TAA 1 resulted in reduced crown root formation, supporting the crucial role of auxin in $A R$ formation in rice (Zhang et al. 2018).

Rice CROWN ROOTLESS 1/ADVENTITIOUS ROOTLESS 1 (CRL1/ARL1), a plant-specific LATERAL ORGAN BOUNDARIES (LOB) family member, is essential for AR initiation. It is auxin-regulated and acts downstream of auxin response factors (ARFs) (Inukai et al. 2005; Liu et al. 2005). A number of additional crown rootless mutants were identified, some of which have been functionally characterized and shown to be involved in auxincontrolled AR initiation and growth. CRL4/OsGNOM1 is a guanine nucleotide exchange ADP-ribosylation factor that controls cycling and polar localization of the auxin efflux carrier PIN1 (Kitomi et al. 2008; Liu et al. 2009). crl4 plants display reduced crown root and lateral root numbers together with impaired gravitropism. CRL5, an AP2/ERF transcription factor, is regulated by auxin through ARF transcription factors. It promotes crown root formation through inhibition of cytokinin signaling (Kitomi et al. 2011). CRL6 encodes a protein from the large chromodomain, helicase/ATPase, and DNA-binding domain (CHD) family. Root initiation and development are under the control by CRL6 through downregulation of OsIAAs, transcriptional repressors of $A R F$ genes in the auxin signaling pathway (Wang et al. 2016). Although CRL2 and CRL3 mutants were shown to have fewer crown roots, their gene functions have yet to be elucidated (Inukai et al. 2001; Kotomi et al. 2008). The auxin efflux carrier protein OsPIN1 mediates auxin-dependent AR emergence. In OsPIN1 RNA interference (RNAi) plants, AR emergence is reduced, a phenotype that is partly reverted by application of the synthetic auxin a-naphthylacetic acid (a-NAA) (Xu et al. 2005). OsPIN: $\beta$-galactosidase reporter gene analysis revealed expression of OsPIN1b and OsPIN1C in growing AR primordia and local expression of OsPIN2 in epidermal cells above AR primordia supporting the idea that polar auxin transport contributes to $A R$ growth and possibly to death of epidermal cells that cover AR primordia which facilitates AR emergence (Steffens and Sauter 2005; Lin and Sauter 2019).

The $\mathrm{Ca}^{2+}$-independent Ser/Thr kinase gene OsRPK1 functions as a negative regulator of AR development by inhibiting polar auxin transport via PIN proteins. Knockdown of OsRPK1 promoted AR initiation (Zou et al. 2014). The Aux/IAA gene OsIAA23 is required for the auxin-mediated maintenance of the quiescent center in postembryonic roots. Osiaa 23 mutants have fewer crown and lateral roots compared with the wildtype (Jun et al. 2011). NARROW LEAF 1 (NAL1) encodes a putative trypsin-like serine/cysteine protease. Loss of NAL1 results in a decreased number of ARs, a phenotype that was rescued by exogenous auxin, suggesting that NAL1 also controls AR growth through the auxin signaling pathway as was shown for several crown rootless mutants (Cho et al. 2013). Moreover, OsCAND1, that is responsible for maintaining the G2/M cell cycle transition, regulates crown root formation through the control of the endogenous auxin level. Oscand 1 shows an abnormal crown root formation that could be partly compensated by exogenous auxin (Wang et al. 2011). Additionally, a point mutation of SQUAMOSA PROMOTER BINDING PROTEIN-LIKE 3 (OSSPL3) repressed the auxin singaling pathway, resulting in a rootless phenotype further supporting the conclusion that auxin plays a crucial role in AR formation in rice (Shao et al. 2019).

\section{Cytokinins}

Growing evidence demonstrates the involvement of cytokinins in AR emergence and growth in rice. Cytokinin receptors activate two types of primary response regulators (RRs), designated type-A and type-B RRs. Type-A RRs act as negative regulators whereas type- $B$ RRs have activating activity. In the presence of cytokinin, type-A RR genes display distinct expression patterns suggestive of specific functions of OsRR proteins in response to cytokinin (Gao et al. 2014). Rice type-A RR6 negatively regulates shoot and root growth. Overexpression of $R R 6$ results in a severe rootless phenotype (Hirose etal.2007).OsCKX4, a cytokinin oxidase/dehydrogenase 
(CKX) family gene, is targeted by auxin response factor OsARF25 and the type-A response regulators OsRR2 and OsRR3. Overexpression of OsCKX4 in rice promotes crown root formation (Gao et al. 2014). Finally, CRL5 is a positive regulator of crown root formation that acts by repressing cytokinin signaling (Kitomi et al. 2011).

The WUSCHEL-related homeobox gene WOX11 is expressed in emerging ARs. It maintains cell division in the root apical meristem. In rice, wox 11 knockout plants and wox11-RNAi lines show reduced crown root formation, whereas overexpression of WOX11 results in ectopic AR growth, indicating that WOX11 is essential for the development of crown root formation in rice (Zhao et al. 2009). The AP2/ERF protein ERF3 interacts with WOX11 and co-regulates crown root development. Knockdown of ERF3 in a wox11 mutant background showed a more severe rootless phenotype whereas overexpression of ERF3 partially rescued the wox 11 phenotype. Both, WOX11 and ERF3 target the type-A RR2 gene that regulates crown root initiation in response to cytokinin (Zhao et al. 2015). WOX11 also recruits an ADA2-GCN5 histone acetyltransferase module to regulate root-specific genes involved in cell division and root meristem activity (Zhou et al. 2017) suggesting that $A R$ formation is also regulated at the epigenetic level. Additional evidence suggests that the TAA/YUC pathway functions together with WOX11, indicating that auxin and cytokinin signaling pathways merge at the level of WOX11 (Zhao et al. 2009; Zhang et al. 2018).

\section{Ethylene and abscisic acid}

Unlike auxin and cytokinin, that are involved in the de novo genesis of rice roots, ethylene represses radicle root growth, and was shown to promote $A R$ emergence and growth in rice. The ethylene signaling pathway of rice remained unexplored for a long time due to the lack of mutants (Rzewuski and Sauter 2008). The transcription factor ETHYLENE INSENSITIVE 3 (EIN3) is a key regulator of ethylene signaling in Arabidopsis. The homologous gene OsEIL1 of rice, when expressed in the Arabidopsis ein3 mutant background, partially compensated the short-root phenotype (Mao et al. 2006) suggesting that key signaling elements are conserved between dicot and monocot plants. Nonetheless, specific regulatory mechanisms in ethylene signaling of root development need yet to be elucidated. The ethylene response factor gene OsERF2 regulates the levels of ethylene and abscisic acid (ABA) and is essential for embryonic root growth (Xiao et al. 2016). In recent years, a number of rice mutants were identified that develop more ARs (Yin et al. 2018). These mutants were termed mao hu zi $(\mathrm{mhz})$, which means 'cat whiskers' (Chen et al. 2018). Several of the corresponding $m h z$ genes were identified through map-based cloning and their functions were studied (Fig. 4A). MHZ7 is the rice homolog of Arabidopsis EIN2 (Merchante et al. 2013; Li et al. 2015). MHZ7:GUS reporter analysis revealed expression of MHZ7 in both the embryonic root and in ARs. Overexpression of MHZ7 led to a short, twisted radicle in rice seedlings (Ma et al. 2013). MHZ6 encodes a homolog of Arabidopsis EIN1. Loss of MHZ6 results in roots that are insensitive to ethylene (Yang et al. 2015). MHZ7 and MHZ6 are considered central components in the ethylene signaling pathway based on the crucial role of orthologous genes EIN2 and EIN1 in Arabidopsis. MHZ3 is an endoplasmic reticulum-localized membrane protein that is required for the stabilization of MHZ7 (Ma et al. 2018). MHZ5 encodes a carotenoid isomerase involved in carotenoid biosynthesis. MHZ5 gene expression was induced by ethylene and results in $A B A$ accumulation in the root, which, in turn, inhibits root growth. MHZ4 is homologous to $A B A 4$ of Arabidopsis, that was previously shown to regulate the interplay between ethylene and $\mathrm{ABA}$. Overexpression of $\mathrm{MHZ4}$ enhanced the ethylene sensitivity of seedling roots and increased the endogenous $A B A$ level. Disruption of $M H Z 4$ induced AR formation at the nodes ( $M a$ et al. 2014). ABA promotes expression of MAIF1 that codes for an F-box domain protein localized at the plasma membrane and in the nucleus. Ectopic overexpression of MAIF1 enhanced root growth (Yan et al. 2011). Taken together, these findings suggest that $M H Z 4$ and $M H Z 5$ mediate the interaction between ethylene and $A B A$ pathways in regulating root growth in rice (Yin et al. 2015). The mutant analysis revealed molecular components of the ethylene-ABA crosstalk that was previously described at the physiological level (Steffens et al. 2006).

\section{Gibberellic acid and salicylic acid}

The hormonal network that regulates AR development and growth in rice includes additional hormones but the molecular mechanisms of their activities have not been exhaustively studied. The AP2/ERF transcription factor $O s S H B$ targets the gene $K S 1$, which encodes the entkaurene synthase of gibberellic acid (GA) biosynthesis 
controlling proliferation and elongation of root meristem cells. Osshb knockout plants have fewer and shorter roots compared with the wildtype (Li et al. 2015) supporting previous physiological studies that revealed enhanced AR growth in the presence of GA (Steffens et al. 2006). The 3-hydroxyacyl-CoA dehydrogenase ABNORMAL INFLORESCENCE MERISTEMI 1 (AIM1) controls root mitotic activity in rice by promotion of salicylic acid (SA) synthesis and repression of redox-related genes and of genes involved in scavenging of reactive oxygen species (ROS), with elevated ROS levels as a result (Xu et al. 2017).

\section{The growth angle of rice ARs is determined by genetic factors and by the environment}

Root system architecture is determined by the number and position of roots, the growth rates and the root growth angles. The functional root system adjusts these parameters to optimize exploitation of the soil and to ensure uptake of nutrients and water in an inhomogeneous soil substrate or when soil parameters change. Analysis of root systems in their natural underground environment is difficult. Non-destructive 3D-imaging techniques such as Magnetic Resonance Imaging (MRI) and X-ray Computer Tomography (CT) elegantly address this issue and allow visualization of entire root systems in soil with a high resolution (Metzner et al. 2015). In general, roots grow toward higher water potential, nutrients and oxygenated areas. By contrast to the soil-borne root system, nodal ARs that emerge upon flooding grow in an aquatic environment where other environmental cues become important.

One such factor is light, which regulates growth of above-ground ARs and of roots that grow at the soil surface. In rice, above-ground ARs grow upward in the dark whereas in the light, ARs grow downward (Lin and Sauter 2018). The AR growth angle is determined by far red, red and blue light revealing negative phototropism controlled by phytochrome ( $\mathrm{Pr}$ and $\mathrm{Pfr}$ ) and blue light signaling likely through phototropin (Lin and Sauter 2018). Roots are sensitive to gravity with primary roots displaying positive gravitropism whereas lateral and ARs grow at an angle that is genetically determined, the gravitropic setpoint angle, and also subject to regulation by environmental cues (Baldwin et al. 2013; Rogers and Benfey 2015). Non-vertical root growth is the result of differential growth rates at opposite sides caused by an asymmetric auxin distribution across the root tip. DEEPER ROOTING 1 (DRO1) is an auxin-regulated gene that promotes a more vertical root growth angle resulting in an overall deeper root system. DRO1 is expressed in the cell elongation zone at the root tip and is responsive to gravity (Uga et al. 2013).

It is well established that polar auxin transport contributes to asymmetric auxin distribution in the root (Petrasek and Friml 2009; Adamowski and Friml 2015). PIN-formed (PIN) proteins are auxin efflux carriers localized at the plasma membrane that are responsible for auxin efflux from the cell. PIN efflux carriers are key determinants of directed auxin transport in the plant and for generating auxin gradients. In Arabidopsis, PIN1 drives acropetal auxin transport in the central cylinder of roots. PIN3, PIN4 and PIN7 redirect the auxin flux in the root cap to the peripheral cell layers while PIN2 directs the basipetal auxin transport toward the root base. PIN5, PIN6 and PIN8 are located at the endoplasmic reticulum and their functions in roots are still largely unknown (Adamowski and Friml 2015). In rice, at least 12 PIN proteins have been predicted based on homologies to Arabidopsis PINs (Fig. 2A). OsPIN1a-d are the rice homologs of AtPIN1 and OsPIN2 is homologous to AtPIN2. AtPIN5 has 3 homologs in rice, OsPIN5a, OsPIN5b and OsPIN5c. OsPIN10a and OsPIN10b are most related to PIN3, PIN4 and PIN7. So far, the functions of OsPINs in determining the root growth angle is largely unclear. The auxin flux mediated by PIN proteins in the rice root can be predicted based on these homologies and the functional characterization of PINs in Arabidopsis (Fig. 2B). OsPINs show different expression patterns in the radicle and in above-ground ARs (Wang et al. 2009; Lin and Sauter 2019). Despite the fact that AR growth direction is largely determined by light, light did not much alter PIN gene expression in ARs. It is conceivable that OsPIN localization and activities are regulated through posttranscriptional mechanisms (Eysholdt-Derzso and Sauter 2017; Lin and Sauter 2019). The OsPIN2 mutant exhibited decreased sensitivity to gravity and showed an increased root growth angle (Wang et al. 2018) suggesting that the gravitropic setpoint angle is in part determined by polar auxin transport via OsPIN2.

In addition to efflux carriers, the auxin influx carrier gene OsAUX1 also positively regulates the root growth 

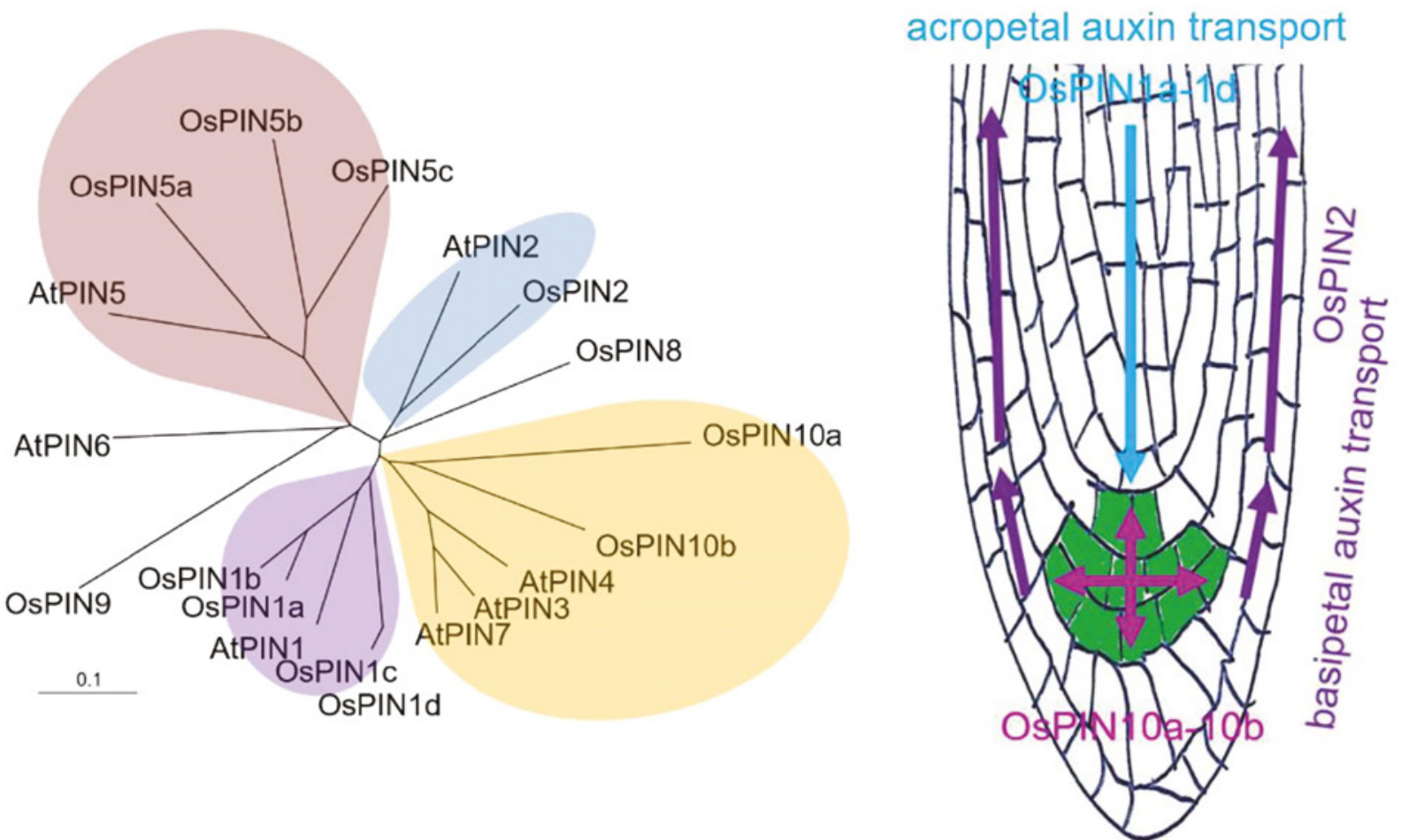

Figure 2. Phylogenetic relationship of PIN proteins in Arabidopsis and rice and predicted polar auxin transport (PAT) in the rice root. (A) Phylogenetic analysis of PIN family members in rice and Arabidopsis. A multiple sequence alignment was carried out with Clustal Omega and a phylogenetic tree was generated using Treeview; bar $=0.1$. (B) Predicted schematic PAT through rice PIN proteins.

angle. Osaux 1 plants have a shallower root system than wild type. In addition, OsAUX1 promotes root hair elongation thereby improving the phosphate acquisition in low phosphate conditions. OsAUX1 plays an important role in auxin transport from the root apex to the differentiation zone (Giri et al. 2018). In summary, investigations of the molecular mechanisms that control the root growth angle in rice have just begun, which holds true also for other cereal crops. Understanding the regulatory network that controls the root growth angle will be a prime task for future research and will help design cereal root systems adapted to a challenging environment.

\section{Changes in root system architecture in response to drought}

Drought causes grain yield loss due to water deficit in the plant (Atkinson and Urwin 2012). Severe or longlasting drought renders the root system damaged or dysfunctional which limits the uptake of water and nutrients or makes it impossible (Rogers and Benfey 2015). For the benefit of the plant and with regard to agricultural yield, an adjustable root system that confers drought tolerance is a desirable trait.

A number of transcription factors have been studied that enhance plant survival under drought conditions. Genes of the NAC domain family of rice have received significant interest due to their specific roles in drought adaptation of the root system (Ooka et al. 2003). Plants that express NAC9 under a root-specific promoter, acquire thicker roots with an enlarged stele and large aerenchyma. Several genes related to root morphogenesis as well as cell elongation and lignin and suberin biosynthesis for walls of the rhizodermis were found to be upregulated in the root of NAC9 overexpressed transgenic plants through microarray analysis (Redillas et al. 2012). The morphological changes might account for the improved grain yield under drought conditions. Similarly, OsNAC10 expression driven by a root-specific promoter caused an increase in root volume, length, dry weight, and diameter together with enhanced 
grain yield, both, in non-stressed and drought conditions (Jeong et al. 2010). SNAC1 is a stress-related NAC family member, that is involved in the adaptation to drought and salt stress. When overexpressed in cotton (disambiguation), SNAC1 produced more roots compared with wildtype plants (Liu et al. 2014). Similarly, NACO45 also confers drought tolerance in rice plants (Zheng et al. 2009), even though the root phenotypes have not been described yet. In summary, NACs confer drought tolerance mainly by increasing the root volume, both, in length and diameter, and by changing the root anatomy. In addition, RSOSPR10, a rice rootspecific pathogenesis-related protein, also enhances drought resistance in plants by enhancing the root mass (Takeuchi et al. 2016). WOX11 mediates drought resistance in plants by controlling the number of crown roots, lateral roots and root hairs (Cheng et al., 2016). In contrast, DEEPER ROOTING 1 (DRO1) increases the root angle allowing the plant to explore water resources in deeper soil layers (Uga et al. 2013; Fig. 3A).

It is well supported that endogenous ABA levels increase in response to drought (Kuromori et al. 2018). ABA alleviates drought stress mainly through enhanced net photosynthesis, stomatal closure and the resulting decreased transpiration rate (Teng et al. 2014). On the other hand, $A B A$ inhibits root growth and hampers water transport. ABA synthesis is mainly detected in young root tips but not in mature roots, which is thought to be an early response to drought (Schachtman and Goodger 2008). However, growing evidence suggests that a high ABA content in longlasting droughts restricts the root growth, which is not beneficial for plants (Shi et al. 2015).

In general, a deep and thick root system confers higher resistance to drought. Thick roots have a larger diameter that enhances the penetration ability in soil allowing faster and deeper growth of roots. In addition, thick roots with additional xylem strands can transport more water, which is beneficial for water uptake and will reduce axial water stress (Yambao et al. 1992). It was estimated that xylem with a pit anatomy is less leaky and transports water more efficiently (Pandey et al. 2015).

During plant breeding, a number of advantageous abiotic stress tolerance genes were lost. It is however possible to identify the respective traits through QTL mapping in the wild rice species. Deeper root is a good example providing the potential for improved rice resistance to drought. The challenge ahead is thus to identify the genes that improve drought tolerance and minimize crop loss.

\section{Adaptative role of rice root system architecture upon flooding}

Flooding and soil water-logging are threatening many crops and weather extremes with severe floods are increasing worldwide (Mustroph 2018). When flooded, the root system is prone to oxygen shortage which limits its uptake and transport capacity for nutrients and water. The maintenance of a functional root system is a prerequisite for plants to survive flooding (Sauter 2013). Several plant species including rice (Lorbiecke and Sauter 1999), tomato (Solanum lycopersicum) and tamarack (Larix laricina) (Calvo-Polanco et al. 2012) developed an adaptive strategy. They replace the damaged roots by ARs through de novo formation or by promoting emergence of existing $A R$ primordia. In rice, ARs are formed at each node during nodal development and remain buried until triggered to emerge by flooding. In tamarack, long-term submergence results in the complete loss of the original roots which are gradually replaced by ARs (Calvo-Polanco et al. 2012). Nodal ARs provide the advantage of a reduced distance for $\mathrm{O}_{2}$ transport from the shoot. The porosity in flooding-induced ARs is higher compared with unflooded roots due to aerenchyma formation (Webb and Jackson 1986; Laan et al. 1989; Drew et al. 2000; Argus et al. 2015). Furthermore, ARs tend to float at or near the water surface which facilitates uptake of atmospheric oxygen (Dawood et al. 2016). In several plants, including Tecticornia pergranulata, aquatic ARs develop chloroplasts and are photosynthetically active to partially compensate for the decreased rate of photosynthesis under water with $\mathrm{O}_{2}$ release as a side effect (Rich et al. 2008). Deepwater rice, adapted to longlasting floods, develops more nodes than lowland rice which allows deepwater rice plants to form more ARs. When floods recede and deepwater rice stems bend to the ground, ARs can grow into the soil thereby avoiding root desiccation and providing anchorage for the plant (Fig. 3A-B).

In rice, induction of $A R$ emergence is triggered by ethylene that gets trapped in the flooded plant (Fig. 4B). Ethylene promotes cell cycle activity at the nodes (Lorbiecke and Sauter 1999), indicating that AR growth is distinctly regulated compared to primary or lateral root growth that is inhibited by ethylene. Inhibition 
A
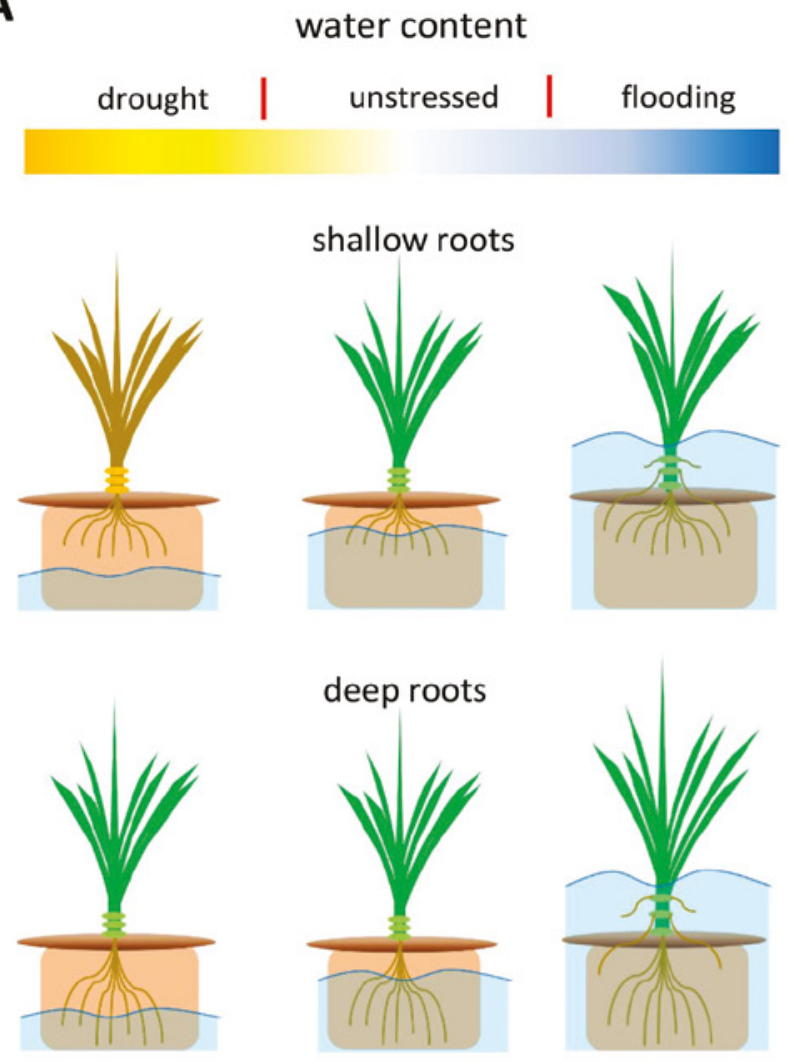

B unflooded control

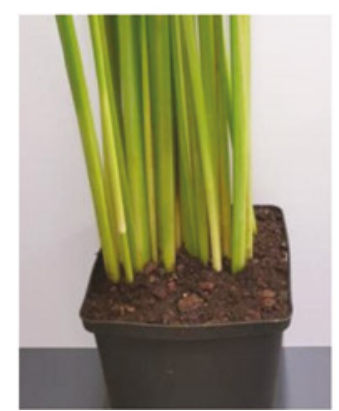

15 days flooding

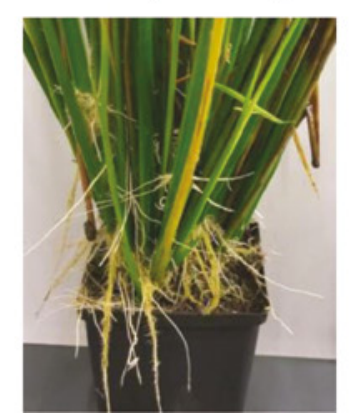

Figure 3. Root systems during drought and flooding. (A) Scheme of a shallow and a deep root system in drought or flooding conditions. (B) Adventitious root growth in 3-month-old Nipponbare plants grown under non-flooded conditions or exposed to partial submergence for 15 days.

of ethylene perception by 1-methylcyclopropene (1$M C P)$ abolished AR emergence revealing a requirement for ethylene signaling (Lin and Sauter 2018). The AR primordium is covered by the nodal epidermis that acts as a physical barrier (Sauter 2013). Programmed death of epidermal cells above AR primordia occurs prior to $A R$ emergence which weakens the epidermal barrier and protects the AR meristem from injury (Steffens and
A

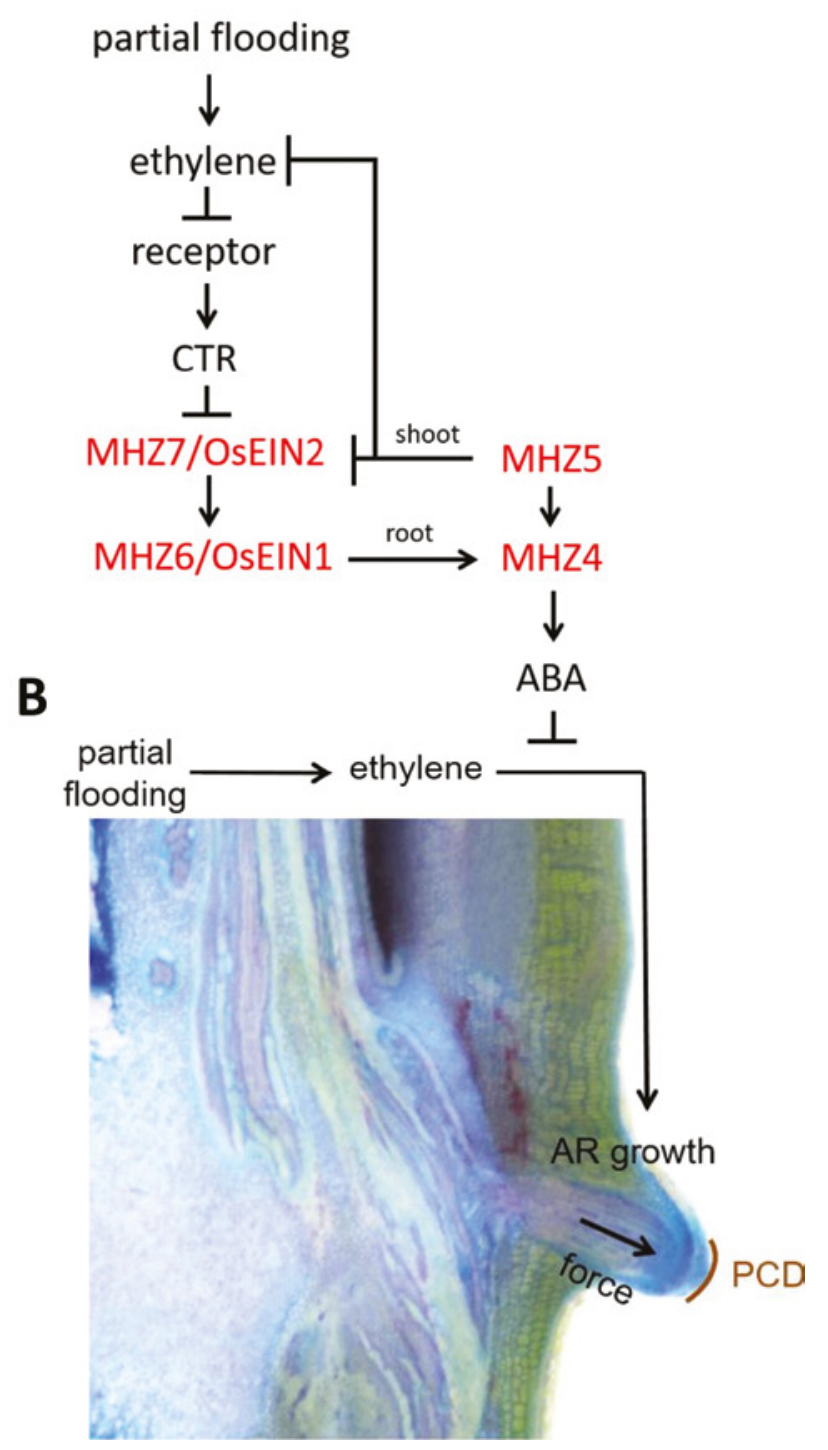

Figure 4. Model of ethylene signaling during submergence. (A) The ethylene signaling pathway of rice based on the analysis of mao hu zi ( $m h z)$ mutants. (B) Ethylene signaling of adventitious root growth and epidermal cell death $(P C D)$ in rice. A nodal cross section was stained with toluidine to visualize the root primordium.

Sauter 2005). Epidermal cell death and AR growth are induced by ethylene, promoted by GA and inhibited by ABA (Steffens et al. 2006) indicating that the interaction and balance of these phytohormones coordinates growth and death in a timely and spatially coordinated manner. Hydrogen peroxide produced by NADPH oxidase at the plasma membrane acts downstream of ethylene in cell death induction. In addition, reduced expression of the ROS scavenger gene METALLOTHIONEIN 
$2 B$ (MT2b) enhances the accumulation of reactive oxygen species and promotes cell death (Steffens and Sauter 2009). The spatial control that limits epidermal cell death to sites where ARs emerge is provided by a mechanical signal coming from the growing AR (Steffens et al. 2012). Both, mechanical force and ethylene signaling via reactive oxygen species are required to trigger the death of epidermal cells.

In rice, ethylene is a positive regulator of AR growth. ARs are crucial for plant survival of submergence. While the blueprint for the ethylene signaling pathway in rice has been established (Fig. 4A) the distinct molecular mechanisms that drive AR growth still need to be uncovered. More so, the majority of studies carried out in rice to date focused on seedlings rather than on mature plants where the adaptive root systems may be controlled differently. And finally, different rice cultivars have developed different survival strategies with an escape response in deepwater rice cultivars and a quiescence response in lowland rice (Voesenek and BaileySerres 2009). It is conceivable that different strategies exist in the development of the AR system. To test this hypothesis, a systematic comparison of root system architecture in different rice types will have to be done.

\section{Conclusion}

Root system architecture in rice is regulated developmentally and by environmental cues. Both regulatory venues rely on phytohormones as key signals. To date, a few genes that are functionally associated with rice root development have been identified and characterized, but the regulatory network that integrates endogenous and exogenous signals still needs to be uncovered. An improved understanding of the molecular mechanisms that control root system development and determine root system architecture will help accelerate breeding of plants with a root system that is optimized to cope with drought and floods to ensure stable crop production.

\section{Disclosure statement}

No conflict of interest by the authors.

\section{Funding}

This scholarship for Chen Lin to study in Germany is granted by Chinese Scholarship Council (CSC).

\section{References}

Adamowski, M, Friml, J. (2015). PIN-dependent auxin transport: action, regulation, and evolution. Plant Cell. 27: 20-32. DOI: 10.1105/tpc.114.134874.

Argus, RE, Colmer, TD, Grierson, PF. (2015). Early physiological flood tolerance is followed by slow post-flooding root recovery in the dryland riparian tree Eucalyptus camaldulensis subsp. refulgens. Plant Cell Environ. 38(6): 1189-99. DOI: 10.1111/ pce.12473.

Atkinson, NJ, Urwin, PE. (2012). The interaction of plant biotic and abiotic stresses: from genes to the field. J. Exp. Bot. 63(10): 3523-43. DOI: 10.1093/jxb/ers100.

Baldwin, KL, Strohm, AK, Masson, PH. (2013). Gravity sensing and signal transduction in vascular plant primary roots. Am. J. Bot. 100: 126-142.

Calvo-Polanco, M, Señorans, J, Zwiazek, JJ. (2012). Role of adventitious roots in water relations of tamarack (Larix laricina) seedlings exposed to flooding. BMC Plant Biol. 12(1): 99. DOI: 10.1186/1471-2229-12-99.

Chen, H, Ma, B, Zhou, Y, He, SJ, Tang, SY, Lu, X, Xie, Q, Chen, SY, Zhang, JS. (2018). E3 ubiquitin ligase SOR1 regulates ethylene response in rice root by modulating stability of Aux/ IAA protein. Proc. Natl. Acad. Sci. USA. 115: 4513-4518. DOI: 10.1073/pnas.1719387115.

Cheng, S, Zhou, DX, Zhao, Y. (2016). WUSCHEL-related homeobox gene WOX 11 increases rice drought resistance by controlling root hair formation and root system development. Plant Signal Behav. 11(2): e1130198. DOI: 10.1080/15592324.2015. 1130198.

Cho, SH, Yoo, SC, Zhang, H, Lim, JH, Paek, NC. (2013). Rice NARROW LEAF1 regulates leaf and adventitious root development. Plant Mol. Biol. Rep. 32: 270-281. DOI: 10.1007/ s11105-013-0675-z.

Coudert, Y, Périn, C, Courtois, B, Khong, NG, Gantet, P. (2010). Genetic control of root development in rice, the model cereal. Trends Plant Sci. 15: 219-226. DOI: 10.1016/j.tplants.2010 .01.008.

Dawood, T, Yang, X, Visser, EJ, Te Beek, TA, Kensche, PR, Cristescu, SM, Lee, S, Floková, K, Nguyen, D, Mariani, C. (2016). A coopted hormonal cascade activates dormant adventitious root primordia upon flooding in Solanum dulcamara. Plant Physiol. 170(4): 2351-64. DOI: 10.1104/pp.15.00773.

Drew, MC, He, CJ, Morgan, PW. (2000). Programmed cell death and aerenchyma formation in roots. Trends Plant Sci. 5(3): 123-7. DOI: 10.1016/S1360-1385(00)01570-3.

Eysholdt-Derzso, E, Sauter, M. (2017). Root bending is antagonistically affected by hypoxia and ERF mediated transcription via auxin signaling. Plant Physiol. 175(1): 412-23. DOl: 10.1104/pp.17.00555.

Gao, S, Fang, J, Xu, F, Wang, W, Sun, X, Chu, J, Cai, B, Feng, Y, Chu, C. (2014). CYTOKININ OXIDASE/DEHYDROGENASE4 integrates cytokinin and auxin signaling to control rice crown root formation. Plant Physiol. 165: 1035-1046. DOI: 10.1104/ pp.114.238584.

Giri, J, Bhosale, R, Huang, G, Pandey, BK, Parker, H, Zappala, S, Yang, J, Dievart, A, Bureau, C, Ljung, K, Price, A. (2018). Rice auxin influx carrier OsAUX1 facilitates root hair elongation 
in response to low external phosphate. Nat. Commun. 9(1): 1408. DOI: 10.1038/s41467-018-03850-4.

Hirose, N, Makita, N, Kojima, M, Kamada-Nobusada, T, Sakakibara, H. (2007) Overexpression of a type-A response regulator alters rice morphology and cytokinin metabolism. Plant Cell Physiol. 48(3): 523-39. DOI: 10.1093/pcp/pcm022.

Inukai, Y, Miwa, M, Nagato, Y, Kitano, H, Yamauchi, A. (2001). Characterization of rice mutants deficient in the formation of crown roots. Breed. Sci. 51(2): 123-129. DOI: 10.1270/ jsbbs.51.123.

Inukai, Y, Sakamoto, T, Ueguchi-Tanaka, M, Shibata, Y, Gomi, K, Umemura, I, Hasegawa, Y, Ashikari, M, Kitano, H, Matsuoka, M. (2005). Crown rootless 1, which is essential for crown root formation in rice, is a target of an AUXIN RESPONSE FACTOR in auxin signaling. Plant Cell. 17(5): 1387-1396. DOI: 10.1105/ tpc.105.030981.

Jeong, JS, Kim, YS, Baek, KH, Jung, H, Ha, SH, Do Choi, Y, Kim, M, Reuzeau, C, Kim, JK. (2010). Root-specific expression of OsNAC10 improves drought tolerance and grain yield in rice under field drought conditions. Plant Physiol. 153(1): 185-197. DOI: 10.1104/pp.110.154773.

Jun, N, Gaohang, W, Zhenxing, Z, Huanhuan, Z, Yunrong, W, Ping, W. (2011). OsIAA23-mediated auxin signaling defines postembryonic maintenance of QC in rice. Plant J. 68(3): 433-442. DOI:10.1111/j.1365-313X.2011.04698.x.

Kitomi, Y, Ito, H, Hobo, T, Aya, K, Kitano, H, Inukai, Y. (2011). The auxin responsive AP2/ERF transcription factor CROWN ROOTLESS5 is involved in crown root initiation in rice through the induction of OSRR1, a type-A response regulator of cytokinin signaling. Plant J. 67(3): 472-484. DOI: 10.1111/j.1365-313X.2011.04610.x.

Kitomi, Y, Kitano, H, Inukai, Y. (2008). Mapping of the CROWN ROOTLESS3 gene, CRL3, in rice. Rice Genet. Newsl. 24: 31-3.

Kitomi, Y, Ogawa, A, Kitano, H, Inukai, Y. (2008). CRL4 regulates crown root formation through auxin transport in rice. Plant Root. 2: 19-28. DOI: 10.3117/plantroot.2.19.

Kuromori, T, Seo, M, Shinozaki, K. (2018). ABA transport and plant water stress responses. Trends Plant Sci. 23(6): 513-522 DOI: 10.1016/j.tplants.2018.04.001.

Laan, P, Berrevoets, MJ, Lythe, S, Armstrong, W, Blom, CW. (1989). Root morphology and aerenchyma formation as indicators of the flood-tolerance of Rumex species. J. Ecol. 1: 693-703. DOI: $10.2307 / 2260979$.

Li, J, Zhao, Y, Chu, H, Wang, L, Fu, Y, Liu, P, Upadhyaya, N, Chen, C, Mou, T, Feng, Y, Kumar, P. (2015). SHOEBOX modulates root meristem size in rice through dose-dependent effects of gibberellins on cell elongation and proliferation. PLoS Genet. 11(8): e1005464. DOI: 10.1371/journal.pgen.1005464.

Li, W, Ma, M, Feng, Y, Li, H, Wang, Y, Ma, Y, Li, M, An, F, Guo, H. (2015). EIN2-directed translational regulation of ethylene signaling in Arabidopsis. Cell. 163(3): 670-83. DOI: 10.1016/j. cell.2015.09.037.

Lin, C, Sauter, M. (2018). Control of adventitious root architecture in rice by darkness, light, and gravity. Plant Physiol. 176(2): 1352-1364. DOI: 10.1104/pp.17.01540.

Lin, C, Sauter, M. (2019). Polar Auxin Transport Determines Adventitious Root Emergence and Growth in Rice. Front. Plant Sci. 10: 444. DOI: 10.3389/fpls.2019.00444.
Liu, G, Li, X, Jin, S, Liu, X, Zhu, L, Nie, Y, Zhang, X. (2014). Overexpression of rice NAC gene SNAC1 improves drought and salt tolerance by enhancing root development and reducing transpiration rate in transgenic cotton. PLoS One. 9(1): e86895. DOI: 10.1371/journal.pone.0086895.

Liu, H, Wang, S, Yu, X, Yu, J, He, X, Zhang, S, Shou, H, Wu, P. (2005). ARL1, a LOB-domain protein required for adventitious root formation in rice. Plant J. 43(1): 47-56. DOI: 10.1111/j.1365-313X.2005.02434.x.

Liu, S, Wang, J, Wang, L, Wang, X, Xue, Y, Wu, P, Shou, H. (2009). Adventitious root formation in rice requires OsGNOM1 and is mediated by the OsPINs family. Cell Res. 19(9): 1110-1119. DOI: 10.1038/cr.2009.70.

Lorbiecke, R, Sauter, M. (1999). Adventitious root growth and cell-cycle induction in deepwater rice. Plant Physiol. 119(1): 21-30. DOI: 10.1111/pce.13094.

Ma, B, He, SJ, Duan, KX, Yin, CC, Chen, H, Yang, C, Xiong, Q, Song, QX, Lu, X, Chen, HW, Zhang, WK. (2013). Identification of rice ethylene-response mutants and characterization of MHZ7/OsEIN2 in distinct ethylene response and yield trait regulation. Mol. Plant 6(6): 1830-1848. DOI: 10.1093/mp/ sst087.

Ma, B, Yin, CC, He, SJ, Lu, X, Zhang, WK, Lu, TG, Chen, SY, Zhang, JS. (2014). Ethylene-induced inhibition of root growth requires abscisic acid function in rice (Oryza sativa L.) seedlings. PLoS Genet. 10(10): e1004701. DOI: 10.1371/journal. pgen.1004701.

Ma, B, Zhou, Y, Chen, H, He, SJ, Huang, YH, Zhao, H, Lu, X, Zhang, WK, Pang, JH, Chen, SY, Zhang, JS. (2018). Membrane protein MHZ3 stabilizes OsEIN2 in rice by interacting with its Nramplike domain. Proc. Natl. Acad. Sci. USA. 115(10): 2520-5. DOI: 10.1073/pnas.1718377115.

Mao, C, Wang, S, Jia, Q, Wu, P. (2006). OsEIL1, a rice homolog of the Arabidopsis EIN3 regulates the ethylene response as a positive component. Plant Mol. Biol. 61(1-2): 141-152. DOI: 10.1007/s11103-005-6184-1.

Mashiguchi, K, Tanaka, K, Sakai, T, Sugawara, S, Kawaide, H, Natsume, M, Hanada, A, Yaeno, T, Shirasu, K, Yao, H, McSteen, P. (2011). The main auxin biosynthesis pathway in Arabidopsis. Proc. Natl. Acad. Sci. USA. 108(45): 18512-7. DOI: 10.1073/ pnas. 1108434108.

Meng, F, Xiang, D, Zhu, J, Li, Y, Mao, C. (2019). Molecular mechanisms of root development in rice. Rice. 12(1): 1. DOI: 10.1186/ s12284-018-0262-x.

Merchante, C, Alonso, JM, Stepanova, AN. (2013). Ethylene signaling: simple ligand, complex regulation. Curr. Opin. Plant Biol. 16(5): 554-60. DOI: 10.1016/j.pbi.2013.08.001.

Metzner, R, Eggert, A, van Dusschoten, D, Pflugfelder, D, Gerth, S, Schurr, U, Uhlmann, N, Jahnke, S. (2015). Direct comparison of MRI and X-ray CT technologies for 3D imaging of root systems in soil: potential and challenges for root trait quantification. Plant Methods 11(1): 17. DOI: 10.1186/s13007-015-0060-z.

Mickelbart, MV, Hasegawa, PM, Bailey-Serres, J. (2015). Genetic mechanisms of abiotic stress tolerance that translate to crop yield stability. Nat. Rev. Genet. 16(4): 237. DOI: 10.1038/ nrg3901.

Mustroph, A. (2018). Improving flooding tolerance of crop plants. Agronomy 8(9):160. DOI: 10.3390/agronomy 8090160 
Ooka, H, Satoh, K, Doi, K, Nagata, T, Otomo, Y, Murakami, K, Matsubara, K, Osato, N, Kawai, J, Carninci, P, Hayashizaki, Y. (2003). Comprehensive analysis of NAC family genes in Oryza sativa and Arabidopsis thaliana. DNA Res. 10(6): 239-47. DOI: 10.1093/dnares/10.6.239.

Orman-Ligeza, B, Parizot, B, Gantet, PP, Beeckman, T, Bennett, MJ, Draye, X. (2013). Post-embryonic root organogenesis in cereals: branching out from model plants. Trends Plant Sci. 18(8): 459-467. DOI: 10.1016/j.tplants.2013.04.010.

Pandey, V, Shukla, A. (2015). Acclimation and tolerance strategies of rice under drought stress. Rice Sci. 22(4): 147-61. DOI: 10.1016/j.rsci.2015.04.001.

Petrasek, J, Friml, J. (2009). Auxin transport routes in plant development. Development. 136(16): 2675-2688. DOI: 10.1242/ dev.030353.

Prosekov, AY, Ivanova, SA. (2018). Food security: The challenge of the present. Geoforum. 91: 73-77. DOI: 10.1016/j. geoforum.2018.02.030.

Rebouillat, J, Dievart, A, Verdeil, JL, Escoute, J, Giese, G, Breitler, JC, Gantet, P, Espeout, S, Guiderdoni, E, Périn, C. (2008). Molecular genetics of rice root development. Rice. 2(1): 15-34. DOI: 10.1007/s12284-008-9016-5.

Redillas, MC, Jeong, JS, Kim, YS, Jung, H, Bang, SW, Choi, YD, Ha, $\mathrm{SH}$, Reuzeau, C, Kim, JK. (2012). The overexpression of OsNAC9 alters the root architecture of rice plants enhancing drought resistance and grainyield underfield conditions. PlantBiotechnol.J. 10(7): 792-805.DOI: 10.1111/j.1467-7652.2012.00697.x.

Rich, SM, Ludwig, M, Colmer, TD. (2008). Photosynthesis in aquatic adventitious roots of the halophytic stemsucculent Tecticornia pergranulata (formerly Halosarcia pergranulata). Plant Cell Environ. 31(7): 1007-1016. DOI: 10.1111/j.1365-3040.2008.01813.x.

Rogers, ED, Benfey, PN. (2015). Regulation of plant root system architecture: implications for crop advancement. Curr. Opin. Biotechnol. 32: 93-98. DOI: 10.1016/j.copbio.2014.11.015.

Rzewuski, G, Sauter, M. (2008). Ethylene biosynthesis and signaling in rice. Plant Sci. 175(1-2): 32-42. DOI: 10.1016/j. plantsci.2008.01.012.

Sauter, M. (2013). Root responses to flooding. Curr. Opin. Biotechnol. 16(3): 282-286. DOI: 10.1016/j.pbi.2013.03.013.

Schachtman, DP, Goodger, JQ. (2008). Chemical root to shoot signaling under drought. Trends Plant Sci. 13(6): 281-287. DOI: 10.1016/j.tplants.2008.04.003.

Shao, Y, Zhou, HZ, Wu, Y, Zhang, H, Lin, J, Jiang, $X, \mathrm{He}, \mathrm{Q}$, Zhu, J, Li, Y, Yu, H, Mao, C. (2019). OsSPL3, an SBP-Domain protein, regulates crown root development in Rice. Plant Cell. 31(6): 1257-75. DOI: 10.1105/tpc.19.00038.

Shi, L, Guo, M, Ye, N, Liu, Y, Liu, R, Xia, Y, Cui, S, Zhang, J. (2015). Reduced $A B A$ accumulation in the root system is caused by ABA exudation in upland rice (Oryza sativa L. var. Gaoshan1) and this enhanced drought adaptation. Plant Cell Physiol. 56(5): 951-64. DOI: 10.1093/pcp/pcv022.

Steffens, B, Kovalev, A, Gorb, SN, Sauter, M. (2012). Emerging roots alter epidermal cell fate through mechanical and reactive oxygen species signaling. Plant Cell. 24(8): 32963306. DOI: 10.1105/tpc.112.101790.
Steffens, B, Sauter, M. (2005). Epidermal cell death in rice is regulated by ethylene, gibberellin, and abscisic acid. Plant Physiol. 139(2): 713-721. DOI: 10.1104/pp.105.064469.

Steffens, B, Sauter, M. (2009). Epidermal cell death in rice is confined to cells with a distinct molecular identity and is mediated by ethylene and $\mathrm{H}_{2} \mathrm{O}_{2}$ through an autoamplified signal pathway. Plant Cell. 21(1): 184-196. DOI: 10.1105/ tpc.108.061887.

Steffens, B, Wang, J, Sauter, M. (2006). Interactions between ethylene, gibberellin and abscisic acid regulate emergence and growth rate of adventitious roots in deepwater rice. Planta. 223(3): 604-612. DOI: 10.1007/s00425-005-0111-1.

Takeuchi, K, Hasegawa, H, Gyohda, A, Komatsu, S, Okamoto, T, Okada, K, Terakawa, T, Koshiba, T. (2016) Overexpression of RSOSPR10, a root-specific rice PR10 gene, confers tolerance against drought stress in rice and drought and salt stresses in bentgrass. Plant Cell Tissue Organ Cult. 127(1): 35-46. DOI: 10.1007/s11240-016-1027-0.

Teng, K, Li, J, Liu, L, Han, Y, Du, Y, Zhang, J, Sun, H, Zhao, Q. (2014). Exogenous $A B A$ induces drought tolerance in upland rice: the role of chloroplast and ABA biosynthesis-related gene expression on photosystem II during PEG stress. Acta Physiol. Plant 36(8): 2219-2227. DOI: 10.1007/s11738-014.

Uga, Y, Sugimoto, K, Ogawa, S, Rane, J, Ishitani, M, Hara, N, Kitomi, Y, Inukai, Y, Ono, K, Kanno, N, Inoue, H. (2013). Control of root system architecture by DEEPER ROOTING 1 increases rice yield under drought conditions. Nature Genet. 45(9): 1097-1102. DOI: 10.1038/ng.2725.

Voesenek, LA, Bailey-Serres, J. (2009). Plant biology: Genetics of high-rise rice. Nature. 460(7258): 959. DOI: 10.1038/460959a.

Wang, JR, Hu, H, Wang, GH, Li, J, Chen, JY, Wu, P. (2009). Expression of PIN genes in rice (Oryza sativa L.): tissue specificity and regulation by hormones. Mol. Plant. 2(4): 823-831. DOI: $10.1093 / \mathrm{mp} / \mathrm{ssp} 023$.

Wang, L, Guo, M, Li, Y, Ruan, W, Mo, X, Wu, Z, Sturrock, CJ, Yu, H, Lu, C, Peng, J, Mao, C. (2018a). LARGE ROOT ANGLE1, encoding OsPIN2, is involved in root system architecture in rice. $J$. Exp. Bot. 69(3): 385-397. DOI: 10.1093/jxb/erx427.

Wang, W, Mauleon, R, Hu, Z, Chebotarov, D, Tai, S, Wu, Z, Li, M, Zheng, T, Fuentes, RR, Zhang, F, Mansueto, L. (2018b). Genomic variation in 3,010 diverse accessions of Asian cultivated rice. Nature. 557(7703): 43. DOI: 10.1038/s41586-018-0063-9.

Wang, XF, He, FF, Ma, XX, Mao, CZ, Hodgman, C, Lu, CG, Wu, P. (2011). OsCAND1 is required for crown root emergence in rice. Mol. Plant. 4(2): 289-299. DOI: 10.1093/mp/ssq068.

Wang, Y, Wang, D, Gan, T, Liu, L, Long, W, Wang, Y, Niu, M, Li, X, Zheng, M, Jiang, L, Wan, J. (2016). CRL6, a member of the CHD protein family, is required for crown root development in rice. Plant Physiol. Biochem. 105: 185-194. DOI: 10.1016/j. plaphy.2016.04.022.

Webb, J, Jackson, MB. (1986). A transmission and cryo-scanning electron microscopy study of the formation of aerenchyma (cortical gas-filled space) in adventitious roots of rice (Oryza sativa). J. Exp. Bot. 37(6): 832-41. DOI: 10.1093/jxb/37.6.832.

Won, C, Shen, X, Mashiguchi, K, Zheng, Z, Dai, X, Cheng, Y, Kasahara, H, Kamiya, Y, Chory, J, Zhao, Y. (2011). Conversion of 
tryptophan to indole-3-acetic acid by TRYPTOPHAN AMINOTRANSFERASES OF ARABIDOPSIS and YUCCAs in Arabidopsis. Proc. Natl. Acad. Sci. USA. 108(45): 18518-23. DOI: 10.1073/ pnas.1108436108.

Xiao, G, Qin, H, Zhou, J, Quan, R, Lu, X, Huang, R, Zhang, $H$. (2016). OsERF2 controls rice root growth and hormone responses through tuning expression of key genes involved in hormone signaling and sucrose metabolism. Plant Mol. Biol. 90(3): 293-302. DOI: 10.1007/s11103-015-0416-9.

Xu, L, Zhao, H, Ruan, W, Deng, M, Wang, F, Peng, J, Luo, J, Chen, Z, $\mathrm{Yi}$, K. (2017). ABNORMAL INFLORESCENCE MERISTEM1 functions in salicylic acid biosynthesis to maintain proper reactive oxygen species levels for root meristem activity in rice. Plant Cell. 29(3): 560-574. DOI: 10.1105/tpc.16.00665.

Xu, M, Zhu, L, Shou, H, Wu, P. (2005). A PIN1 family gene, OsPIN1, involved in auxin-dependent adventitious root emergence and tillering in rice. Plant Cell Physiol. 46(10): 1674-1681. DOI: 10.1093/pcp/pci183.

Yamamoto, Y, Kamiya, N, Morinaka, Y, Matsuoka, M, Sazuka, T. (2007). Auxin biosynthesis by the YUCCA genes in rice. Plant Physiol. 143(3): 1362-1371. DOI: 10.1104/pp.106.091561.

Yambao, EB, Ingram, KT, Real, JG. (1992). Root xylem influence on the water relations and drought resistance of rice. J. Exp. Bot. 43(7): 925-932. DOI: 10.1093/jxb/43.7.925.

Yan, YS, Chen, XY, Yang, K, Sun, ZX, Fu, YP, Zhang, YM, Fang, RX. (2011). Overexpression of an F-box protein gene reduces abiotic stress tolerance and promotes root growth in rice. Mol. Plant. 4(1): 190-197. DIO: 10.1093/mp/ssq066.

Yang, C, Ma, B, He, SJ, Xiong, Q, Duan, KX, Yin, CC, Chen, H, Lu, $X$, Chen, SY, Zhang, JS. (2015). MAOHUZI6/ETHYLENE INSENSITIVE3-LIKE1 and ETHYLENE INSENSITIVE3-LIKE2 regulate ethylene response of roots and coleoptiles and negatively affect salt tolerance in rice. Plant Physiol. 169(1): 148-165. DOI: 10.1104/pp.15.00353.

Yin, CC, Ma, B, Collinge, DP, Pogson, BJ, He, SJ, Xiong, Q, Duan, KX, Chen, H, Yang, C, Lu, X, Wang, YQ. (2015). Ethylene responses in rice roots and coleoptiles are differentially regulated by a carotenoid isomerase-mediated abscisic acid pathway. Plant Cell. 27(4): 1061-1081. DOI: 10.1105/tpc.15.0008.

Yin, CC, Ma, B, Zhao, H, Chen, SY, Zhang, JS. (2018). Screening and genetic analysis of ethylene-response mutants in etiolated rice seedlings. Bio-Protocol. DOI:10.21769/BioProtoc.3001.

Zhang, T, Li, R, Xing, J, Yan, L, Wang, R, Zhao, Y. (2018). The YUCCA-Auxin-WOX11 module controls crown root development in rice. Front. Plant Sci. 9: 523. DOI: 10.3389/fpls. 2018.00523.

Zhao, Y. (2012). Auxin biosynthesis: a simple two-step pathway converts tryptophan to indole-3-acetic acid in plants. Mol. Plant. 5(2): 334-338. DOI: 10.1093/mp/ssr 104.

Zhao, Y,Cheng, S, Song, Y, Huang,Y,Zhou, S, Liu, X,Zhou, DX. (2015). The interaction between Rice ERF3 and WOX11 promotes crown root development by regulating gene expression involved in cytokinin signaling. Plant Cell. 27(9): 2469-2483. DOI: 10.1105/tpc.15.00227.

Zhao, Y, Hu, Y, Dai, M, Huang, L, Zhou, DX. (2009). The WUSCHELrelated homeobox gene WOX 11 is required to activate shootborne crown root development in rice. Plant Cell. 21(3): 736-748. DOI: 10.1105/tpc.108.061655.

Zheng, X, Chen, B, Lu, G, Han, B. (2009). Overexpression of a NAC transcription factor enhances rice drought and salt tolerance. Biochem. Biophys. Res. Commun. 379(4): 985-989. DOI: 10.1016/j.bbrc.2008.12.163.

Zhou, S, Jiang, W, Long, F, Cheng, S, Yang, W, Zhao, Y, Zhou, DX. (2017). Rice homeodomain protein WOX11 recruits a histone acetyltransferase complex to establish programs of cell proliferation of crown root meristem. Plant Cell. 29(5): 1088-1104. DOI: 10.1105/tpc.16.00908.

Zou, Y, Liu, X, Wang, Q, Chen, Y, Liu, C, Qiu, Y, Zhang, W. (2014). OsRPK1, a novel leucine-rich repeat receptor-like kinase, negatively regulates polar auxin transport and root development in rice. Biochim. Biophys. Acta. Gen. Subj. 1840(6): 1676-85. DOI: 10.1016/j.bbagen.2014.01.003. 